\title{
Controlled antiseptic release by alginate polymer films and beads
}

\author{
Ioannis Liakos ${ }^{a}, *$, Loris Rizzello ${ }^{a}$, Ilker S. Bayer ${ }^{a}, * *$, Pier Paolo Pompa ${ }^{a}$, Roberto Cingolani ${ }^{b}$, \\ Athanassia Athanassiou ${ }^{\mathrm{a}, \mathrm{b}, * * *}$ \\ a Center for Biomolecular Nanotechnologies, Istituto Italiano di Tecnologia @UniLe, via Barsanti, 73010 Arnesano, Lecce, Italy \\ b Istituto Italiano di Tecnologia (IIT), via Morego 30, 16163 Genova, Italy
}

\section{A R T I C L E I N F O}

\section{Article history:}

Received 4 August 2012

Received in revised form 31 August 2012

Accepted 15 September 2012

Available online 27 September 2012

\section{Keywords:}

Sodium and calcium alginate

Povidone iodine

Wound dressing

Drug encapsulation

Drug release

\begin{abstract}
A B S T R A C T
Biodegradable polymeric materials based on blending aqueous dispersions of natural polymer sodium alginate (NaAlg) and povidone iodine (PVPI) complex, which allow controlled antiseptic release, are presented. The developed materials are either free standing NaAlg films or $\mathrm{Ca}^{2+}$-cross-linked alginate beads, which properly combined with PVPI demonstrate antibacterial and antifungal activity, suitable for therapeutic applications, such as wound dressing. Glycerol was used as the plasticizing agent. Film morphology was studied by optical and atomic force microscopy. It was found that PVPI complex forms well dispersed circular micro-domains within the NaAlg matrix. The beads were fabricated by drop-wise immersion of NaAlg/PVPI/glycerol solutions into aqueous calcium chloride solutions to form calcium alginate beads encapsulating PVPI solution (CaAlg/PVPI). Controlled release of PVPI was possible when the composite films and beads were brought into direct contact with water or with moist media. Bactericidal and fungicidal properties of the materials were tested against Escherichia coli bacteria and Candida albicans fungi. The results indicated very efficient antibacterial and antifungal activity within $48 \mathrm{~h}$. Controlled release of PVPI into open wounds is highly desired in clinical applications to avoid toxic doses of iodine absorption by the wound. A wide variety of applications are envisioned such as external and internal wound dressings with controlled antiseptic release, hygienic and protective packaging films for medical devices, and polymer beads as water disinfectants.
\end{abstract}

(C) 2012 Elsevier Ltd. All rights reserved.

\section{Introduction}

Naturally occurring alginate polymers have long been used in the food and beverage industries as thickening, gel-forming and colloidal stabilizing agents (Donati \& Paoletti, 2009; Moe, Draget, Skjak-Bræk, \& Smidsrod, 1995). They are also used in the pharmaceutical industry as matrices for drug encapsulation, as substrates for cell culture, as binders for medical tablets (Goh, Heng, \& Chan, 2012) and for many applications of controlled transdermal or transmucosal drug delivery of active substances (Sachan, Pushkar, Jha, \& Bhattcharya, 2009). Commercial alginates are extracted primarily from marine algae such as Laminaria hyperborea, Ascophyllum nodosum and Macrocystis pyrifera (Wee \& Gombotz, 1998). Alginates are linear unbranched polysaccharides containing varying proportions of beta-D-mannuronate (M) and alpha-L-guluronate

\footnotetext{
* Corresponding author. Tel.: +390832 295705.

** Corresponding author.

$* * *$ Corresponding author at: Istituto Italiano di Tecnologia, Nanophysics, via Morego 30, 16163 Genova, Italy.

E-mail addresses: ioannis.liakos@iit.it (I. Liakos), ilker.bayer@iit.it (I.S. Bayer), athanassia.athanassiou@iit.it (A. Athanassiou).
}

(G) residues. Molecular variability in this polymer depends on the source of marine algae, tissue from which alginates are extracted, and also the season of crop harvesting. The composition, sequence of polymer blocks and molecular weight of alginates are important as these factors determine the physical properties of the gel formed. In solution, alginates behave like flexible coils, but when they interact with calcium ions (such as in a calcium chloride solution) they form ordered structures. In fact, cross linking of water soluble sodium alginate can be initiated by polyvalent cations such as calcium, forming water insoluble calcium alginate. The mechanism of CaAlg bead formation involves cooperative binding of calcium ions between two aligned GG blocks of two alginate chains. The formation of CaAlg beads is an instantaneous and irreversible process, which is determined by the rate of diffusion of calcium ions in the sodium alginate polymer matrix (Blandino, Macias, \& Cantero, 1999).

Recent trends in biomedical and pharmaceutical industry indicate that alginates (Goh et al., 2012; Shilpa, Agrawal, \& Ray, 2003), are increasingly explored for the development of new drug delivery materials. Among alginates, sodium alginate ( $\mathrm{NaAlg}$ ) is the most widely explored. Being non-toxic hydrophilic and biodegradable, it can be absorbed from skin into the body fluids without any toxic effects (Sachan et al., 2009), ideal characteristics for the 
wound healing process (Chiu, Lee, Chu, Chang, \& Wang, 2008). It has been reported that the potential combination with antimicrobial and enzymatic components can promote elimination of necrotic tissues and microbial bodies, while the polysaccharide base can stimulate reparative wound process (Patel et al., 2007). Chitosan is another natural marine polymer extracted from sea crustaceous with exceptional properties (Paños, Acosta, \& Heras, 2008; Spano, Massaro, Blasi, Malerba, \& Cingolani, 2012). The combination of NaAlg with chitosan has become quite commonplace for the development of potential wound healing materials (films, and membranes) as they showed no toxic effects to mammalian cells (Wang, Khor, Wee, \& Yong Lim, 2002). A very recent study has shown that $\mathrm{NaAlg} /$ chitosan-based wound dressing films in combination with a special laser treatment therapy significantly accelerated burn healing by modulating the epithelization, blood vessels formation and collagenization process (Dantas et al., 2011). Extract of plants that have wound healing properties have also been incorporated into alginate films. Asiaticoside, a component derived from Centella asiatica Linn., has been used with alginate to produce films to heal wounds, burns and ulcer abnormalities on the skin (Sikareepaisana, Ruktanonchai, \& Supaphol, 2011). Alginate and alginate/chitosan microcapsules with controlled drying properties have shown to increase the viability of the encapsulated probiotic Bifidobacterium breve during exposure at stimulated gastric fluid (Cook, Tzortzis, Charalampopoulos, \& Khutoryanskiy, 2011).

In this work, we present fabrication and characterization of alternative and highly effective antibacterial and antifungal biomaterials combing sodium and calcium alginates with a traditional antiseptic, the povidone iodine complex (PVPI, or betadine). PVPI is a water soluble complex of iodine and polyvinylpyrrolidone, widely used for its bactericidal and fungicidal properties. PVPI is in fact an iodophor. Iodophors are compounds of iodine linked to surfactants that act as carriers or solubilizing agents. As such, a small amount of free iodine is released in solution, thereby minimizing toxicity while preserving moderate germicidal activity of the element (Heiner, Hile, Demons, \& Wedmore, 2010). The most common form of PVPI complex is its aqueous solution available in squeeze bottles containing from 9 to $12 \mathrm{wt} \%$ available iodine, which can be purchased off the shelf without prescription. Aqueous PVPI solutions are directly applied on open wounds or infected regions, a process commonly known as wound irrigation. Although slow release of iodine from the PVPI complex in solution minimizes iodine toxicity toward mammalian cells, its sudden absorption by some open wounds during irrigation can cause severe toxicity problems and sever skin burn limiting its surgical applications, particularly under repeated applications (Nahlielia, Baruchinb, Levia, Shapiraa, \&Yoffea, 2001; Wong et al., 2011). In addition to its wide use for skin antisepsis, PVPI is also employed internally. For instance, it is often applied on large internal wounds, deep tissues or mucosa (Lakhal, Faidherbe, Choukhi, Boissier, \& Capdevila, 2011). Due to the ability of polyvinylpyrrolidone polymer to absorb water up to $40 \%$ of its weight, PVPI complex films easily lose their consistency or wash away quickly in highly humid or water containing media. In such cases their antibacterial effect is drastically reduced. To avoid this effect, recent studies propose, for instance, immobilization of PVPI complex on polymer surfaces on single-walled carbon nanotube surfaces (Simmons et al., 2009) or incorporation into hydrogels (Mishra \& Chaudhary, 2010). We demonstrate a straightforward way to prepare dry NaAlg/PVPI composite films as potential wound dressing materials by simple solution casting from water suspensions, with the ability of controlled release of PVPI into liquid media. Alternatively, by dripping small successive quantities of aqueous solutions of NaAlg and PVPI into a $\mathrm{CaCl}_{2}$ water solution through a syringe, PVPI solution can be encapsulated in calcium cross linked alginate beads, to enable longer lasting controlled release of PVPI
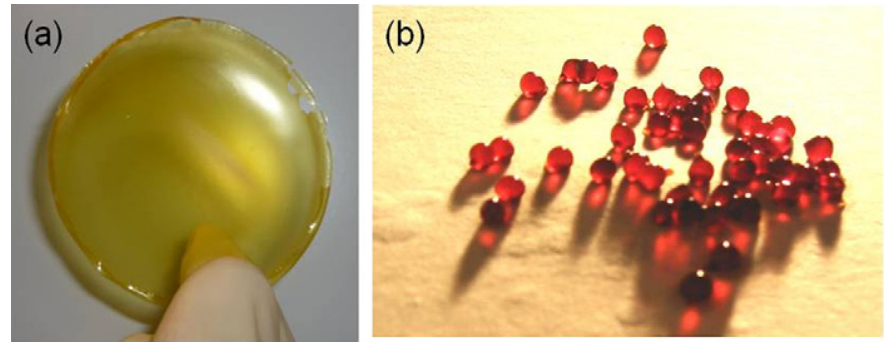

Fig. 1. Photographs of (a) NaAlg/PVPI complex composite film plasticized with glycerol and (b) CaAlg beads encapsulating PVPI complex liquid.

into liquid media. Both NaAlg/PVPI films and CaAlg/PVPI beads showed highly efficient antimicrobial and antifungal properties against Escherichia coli bacteria and Candida albicans fungi. These films and beads can be easily adapted to large area dry wounds as dressing, sterile biodegradable surface coatings and to internal wound treatment for slow drug release (Athanassiou et al., 2012).

\section{Material and method}

\subsection{Materials}

Alginic acid sodium salt with viscosity 15,000-20,000 centipoises (cps), glycerol $\geq 99.5$ (GC) and calcium chloride anhydrous were purchased from Sigma-Aldrich. PVPI, $100 \mathrm{ml}$ solution in water contained $10 \mathrm{wt} \%$ PVPI of which $1 \mathrm{wt} \%$ was active iodine, named as LH IODO 10, was purchased from Pharmatek PMC, Italy.

\subsection{Films preparation}

Three different NaAlg-PVPI blend films were studied herein. Films were cast from three identical NaAlg in water stock solutions containing different amounts of PVPI solution with a total volume of $10 \mathrm{ml}$. More specifically, distilled water and $0.3 \mathrm{~g}$ of NaAlg were mixed together and warmed-up using a hot plate for $1 \mathrm{~h}$ near $100^{\circ} \mathrm{C}$ to ensure complete dissolution of the alginate in water. Afterwards, the viscous solutions were left to cool to room temperature and to these $0.1 \mathrm{ml}$ of glycerol was added as a plasticizer. The amount of added glycerol was kept constant in all the subsequent solution preparations. Next, $1.0,1.5$ and $2.0 \mathrm{ml}$ of aqueous PVPI solution were slowly blended into the NaAlg/glycerol solutions. The final solutions were mixed using a vortex mixer and left for a while to ensure that no bubbles were present in the vials. For the films' formation, $200 \mu \mathrm{l}$ of the prepared solutions were drop cast on glass slides of $1 \mathrm{~cm} \times 1 \mathrm{~cm}$ area and left under ambient laboratory conditions for drying. The resultant dry films obtained from these solutions have the following composition: Film 1: 57\% NaAlg, 24\% glycerol and 19\% PVPI; Film 2: 52\% NaAlg, 22\% glycerol and 26\% PVPI; Film 3: 48\% NaAlg, 20\% glycerol and 32\% PVPI. According to the manufacturer, the specific weight of PVPI polymer is approximately 1.03. Therefore, for all practical purposes, the density of PVPI was taken as $1.0 \mathrm{~g} / \mathrm{ml}$ for dry film composition calculations. The ratio of NaAlg to glycerol in all the films was between 2.3 and 2.4. Fig. 1a shows a photograph of a representative flexible biodegradable NaAlg/PVPI film that was previously cast on a plastic Petri dish. Samples for Fourier transform infrared (FTIR) spectroscopy analysis were instead prepared by spin coating of $1 \mathrm{ml}$ of the above described solutions (in 2 steps: $500 \mathrm{rpm}$ for $10 \mathrm{~s}$ and $750 \mathrm{rpm}$ for $30 \mathrm{~s})$ on silicon wafers and then slightly baked on a hot plate $\left(60^{\circ} \mathrm{C}\right.$ for $1-2 \mathrm{~min}$ ) to obtain thin films and avoid saturation effects during the infrared spectroscopy analysis. 


\subsection{Beads preparation}

The above mentioned solution used in the fabrication of Film 3 (48\% NaAlg, 20\% glycerol and 32\% PVPI), was chosen to prepare $\mathrm{CaAlg} / \mathrm{PVPI}$ beads. The NaAlg/PVPI/glycerol solution was drop-wise immersed into a $10 \% \mathrm{CaCl}_{2}$ solution in distilled water. The formation of the beads was immediate. Subsequently, the beads were collected and rinsed with distilled water to remove excess $\mathrm{CaCl}_{2}$ traces. Fig. 1b shows a photograph of CaAlg/PVPI beads collected from the $\mathrm{CaCl}_{2}$ solution.

\section{Experimental}

\subsection{Optical and atomic force microscopy}

Optical microscopy images were acquired with a Zeiss AXIO instrument. For atomic force microscopy (AFM) images, a Park System AFM instrument (XE-100) was used in true non-contact mode, on an anti-vibration table (Table Stable TS-150) and within an acoustic enclosure. Single-beam silicon cantilevers tips (PPPNCHR-10) were used for the data acquisition with about less than $10 \mathrm{~nm}$ nominal radius and $42 \mathrm{~N} / \mathrm{m}$ elastic force constant for high sensitivity. The resonance frequency was defined around $280 \mathrm{kHz}$. The scan rate was between 0.2 and $1.0 \mathrm{~Hz}$.

\subsection{Fourier transform infrared (FTIR) spectroscopy}

FTIR analyses were performed with a VERTEX 70 FTIR instrument in the spectral range from 4000 to $400 \mathrm{~cm}^{-1}$ in absorbance mode.

\subsection{Measurement of PVPI release from NaAlg films and CaAlg beads}

Film 1 and Film 3 were chosen for the measurement of PVPI release into distilled water. Small samples cut from the films were placed at the bottom of UV cuvettes, which were then positioned in a CARY 300 Scan UV-visible spectrophotometer from Varian. The cuvettes were filled with $2.5 \mathrm{ml}$ distilled water and the release of PVPI was monitored in time by following the increase of the PVPI characteristic UV-vis absorption peaks. Gentle agitation of the cuvette before each UV-vis absorption measurement step was necessary in order to disperse the released PVPI evenly in the cuvette.

In order to measure the release of PVPI solution from freshly prepared CaAlg beads, $0.5 \mathrm{ml}$ stock solution used to make Film 3 was converted into beads by drop-wise immersion into $\mathrm{CaCl}_{2}$ solution. After collection and rinsing, the beads were added into the cuvette containing $2.5 \mathrm{ml}$ distilled water and the characteristic UV-vis absorption peak of PVPI was monitored in time as described above.

\subsection{Antibacterial/antifungal activity of NaAlg/PVPI films and CaAlg/PVPI beads}

In these experiments, the activity of $\mathrm{NaAlg} / \mathrm{PVPI}$ films coated on glass slides and CaAlg/PVPI beads was tested against two different organisms, namely E. coli, a model Gram-negative bacterium, and $C$. albicans, a fungus which is the causal agent of many opportunistic oral and genital infections in humans.

A loop of glycerol stock of $E$. coli strain TG1 was streaked onto a Luria-Bertani (LB) medium agar plate and incubated overnight at $37^{\circ} \mathrm{C}$. Then a single colony was picked up and grown in LB liquid medium overnight at $37^{\circ} \mathrm{C}$ to reach a bacterial concentration of $\sim 8 \times 10^{8}$ cells $/ \mathrm{ml}$, in a shaking incubator. To prepare the fungal cell suspension, two to three $C$. albicans colonies were picked from the plate, washed in isotonic glucose phosphate buffer (IGP; $1 \mathrm{mM}$
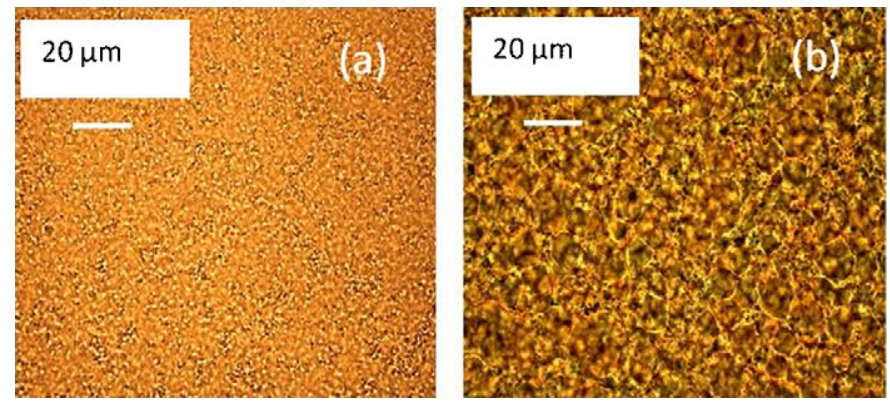

Fig. 2. (a) Representative optical microscopy image showing the morphology of $70 \mathrm{wt} \% \mathrm{NaAlg}$ and $30 \mathrm{wt} \%$ glycerol dry films, drop cast from a water solution. (b) Representative optical microscopy image showing the morphology of Films 3 (48\% NaAlg, 20\% glycerol and 32\% PVPI). Micro-phase separation is evident from the appearance of micro-circular features throughout the sample.

potassium phosphate buffer, pH 7.0, supplemented with $287 \mathrm{mM}$ glucose as an osmoprotectant), and a fungal cell suspension was prepared by adjusting the inoculum to $10^{8}$ cells $/ \mathrm{ml}$.

To assess the antibacterial behavior of NaAlg/PVPI films, the overnight $E$. coli culture was diluted to a final concentration of $10^{6}$ cells $/ \mathrm{ml}$, and $500 \mu \mathrm{l}$ of this solution was spread onto new LB medium agar plates. The plates were then placed in the incubator at $37^{\circ} \mathrm{C}$ for $2 \mathrm{~h}$ (to allow a proper evaporation of residual liquid medium), followed by the positioning of the different substrates ( 2 for each LB plates). To investigate the antifungal activity of NaAlg/PVPI films, the initial $10^{8}$ cells $/ \mathrm{ml}$ inoculum was diluted to $10^{5}$ cells/ml and $100 \mu \mathrm{l}$ of this solution was spread onto freshly prepared Sabouraud dextrose agar (SDA). The plates were then incubated at $37^{\circ} \mathrm{C}$ for $2 \mathrm{~h}$, followed by the positioning of the different substrates ( 2 for each plate). The substrates were overnight sterilized, by UV irradiation, inside a biohazard hood before their use.

The antibacterial/antifungal behavior of CaAlg/PVPI beads was tested by turbidity assay. In particular, a confluent overnight $C$. albicans culture was diluted to an optical density $\left(\mathrm{OD}_{600}\right)$ of 0.1 , then $1 \mathrm{~g}$ of $\mathrm{CaAlg} / \mathrm{PVPI}$ beads was added to the culture and the optical density, at $600 \mathrm{~nm}$ the commonly used wavelength for cell growth evaluation, was measured every $30 \mathrm{~min}$ with a spectrophotometer (Thermo Scientific). The same turbidity assays were performed with $E$. coli, but in this case $12 \mathrm{~g}$ of CaAlg/PVPI beads were supplemented to the bacteria culture. All the beads used for the experiments were previously sterilized by an overnight UV irradiation inside a biohazard hood.

\section{Results and discussion}

\subsection{Investigation of the NaAlg/PVPI films morphology with optical and atomic force microscopy}

Fig. 2(a) illustrates a representative optical microscope image showing the morphology of a glycerol plasticized NaAlg dry film made up of $70 w t \% ~ N a A l g$ and $30 w t \%$ glycerol. Fig. 2(b), on the other hand, shows the optical microscope image of a dry Film $3(48 \%$ NaAlg, 20\% glycerol and 32\% PVPI). Careful inspection of Fig. 2(b) reveals the existence of circular domains with 10-20 $\mu$ m diameter throughout the composite film. This is a first indication that PVPI and NaAlg are not fully miscible, and micro-phase separation occurs during drying of the composite films.

Fig. 3 shows images of AFM surface topography and phase contrast of different drop cast films. The films were drop cast on thin glass slides from their respective stock solutions. The dry film shown in Fig. 3(a) contains 100 wt\% NaAlg, whereas the dry film in Fig. 3(b) contains $70 \mathrm{wt} \% \mathrm{NaAlg}$ and $30 \mathrm{wt} \%$ glycerol. Glycerol is 

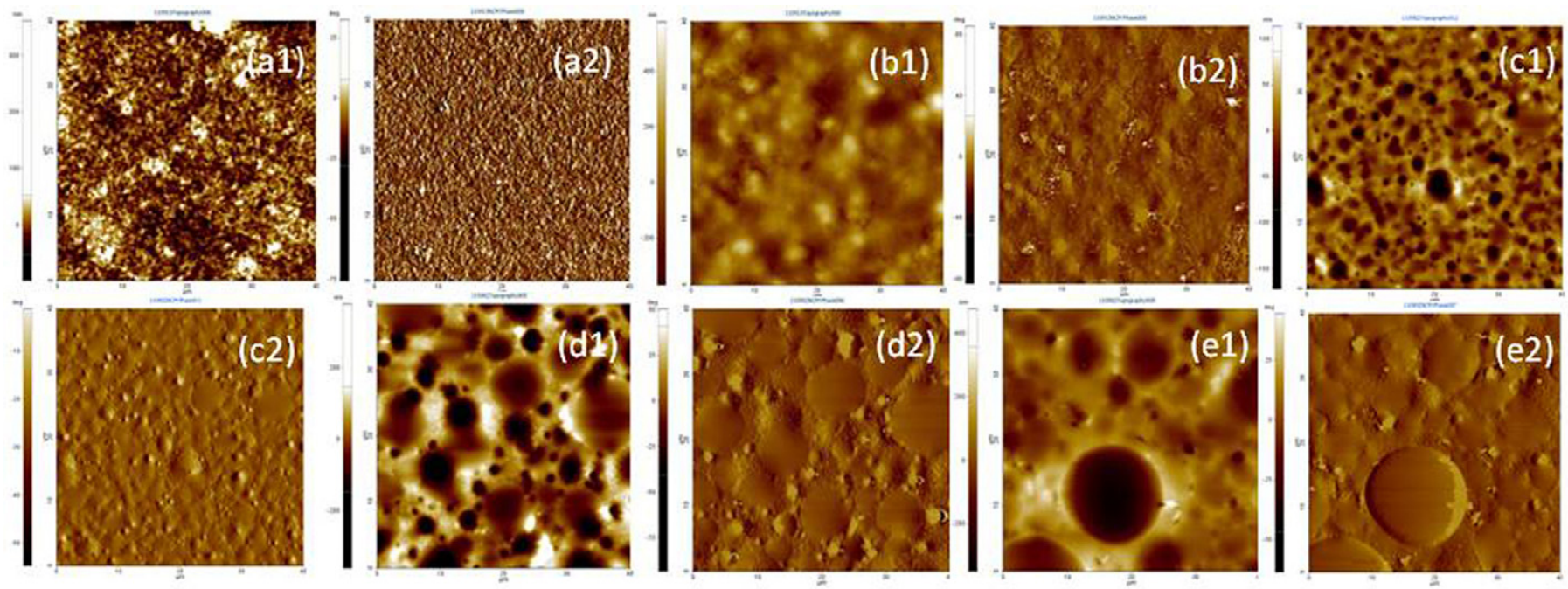

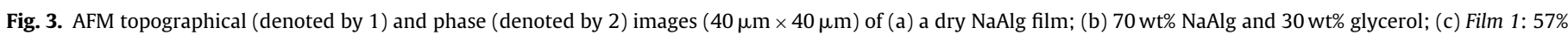
NaAlg, 24\% glycerol and 19\% PVPI; (d) Film 2: 52\% NaAlg, 22\% glycerol and 26\% PVPI and (e) Film 3:48\% NaAlg, 20\% glycerol and 32\% PVPI.

a low-molecular weight nonvolatile substance and is a common plasticizer for natural polymers and pharmaceutical materials. It was shown to reduce internal hydrogen bonding between polymer chains while increasing molecular volume, which results in film flexibility (Maizura, Fazilah, Norziah, \& Karim, 2007). Maizura et al. also demonstrated that NaAlg films plasticized by glycerol show highly smooth and defect (crack) free surface morphology, whereas pure solution cast NaAlg films can form cracks at times reducing their mechanical properties as well as increasing their water vapor permeability. Moreover, the Young's modulus of NaAlg films is reduced and their percent elongation at break is increased with the incorporation of glycerol (Russo, Malinconico, Petti, \& Romano, 2005), which is in accordance with the Dynamic Mechanical Analysis measurements conducted on our films (not shown here). Morphological and phase AFM analysis results from the PVPIincorporating Films 1, 2 and 3 are shown in Fig. 3(c, d and e), respectively. Various sizes of micro-circular domains attributed to PVPI are clearly seen in the composite structures. It is also observed that for increasing PVPI concentration the circular domains inside the NaAlg-glycerol matrix become larger. From the phase images, it is clear that the PVPI circular domains are well dispersed and separated from the matrix.

The results are in accordance with a recent work (Caykara, Demirci, Eroglu, \& Guven, 2006) on NaAlg/polyvinylpyrrolidone (PVP) (without iodine) blend films. Various AFM results from this study also indicated phase separation in the form of circular PVP micro domains in NaAlg films. Their analysis based on acid-base adhesion theory indicated that the surface free energy of PVP has a basic character whereas the surface free energy of NaAlg is somewhat acidic. This led to the conclusion that when the surface acidity of one component is high while the surface basicity of the other component is also high, the two components show good compatibility, though not miscible, caused by strong adhesion between them, even if micro-phase separation takes place.

\subsection{Fourier transform infrared spectroscopy}

In Fig. 4, the FTIR spectra of all the fabricated films obtained in the range $400-4000 \mathrm{~cm}^{-1}$ wave numbers are shown. The main peak, common in all the films, at $3380 \mathrm{~cm}^{-1}$ is attributed to the $\mathrm{OH}$ stretching, which is readily present in the alginic acid backbone of NaAlg, and to the presence of $\left(\mathrm{O} \cdots \mathrm{H}^{+} \ldots \mathrm{O}\right)$ bridges within the PVPI complex film. In all the samples, there is also a peak at $2943 \mathrm{~cm}^{-1}$, which is attributed to $\mathrm{CH}_{2}$ stretching. The peak at $2890 \mathrm{~cm}^{-1}$ appears in samples plasticized with Glycerol, corresponding to spectra denoted by (1) to (5), and is attributed to $\mathrm{CH}$ band stretching. The same peak is also present in the PVPI spectrum, denoted by spectra (6), and originates from the PVP backbone. Detailed inspection of spectra also indicates existence of a shoulder at $1463 \mathrm{~cm}^{-1}$, which is present in samples plasticized with glycerol and can be attributed to the $\mathrm{C}-\mathrm{O}$ band stretching of the primary alcohol. The other clearly distinguished peaks appearing in all the six samples are the anti-symmetric carboxylate band stretching centered around $1596 \mathrm{~cm}^{-1}$ and the symmetric carboxylate band stretching around $1420 \mathrm{~cm}^{-1}$, and were also observed in previous studies on NaAlg polymers (Lawrie et al., 2007; Sartori, Finch, Ralph, \& Gilding, 1997). In addition, the peak at $1665 \mathrm{~cm}^{-1}$ was only present in the composites with PVPI, namely the spectra denoted

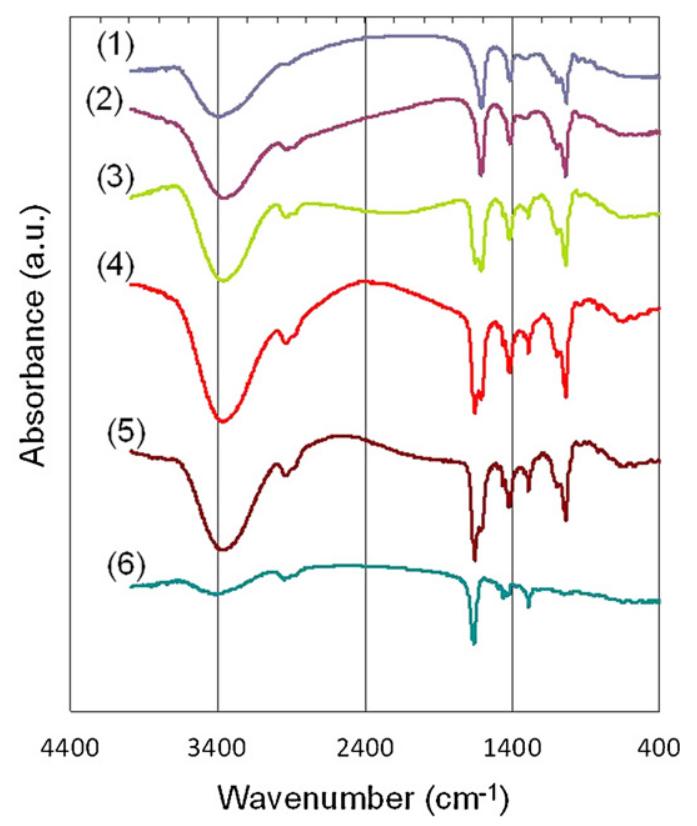

Fig. 4. FTIR spectra of all the films studied: (1) $100 \mathrm{wt} \% \mathrm{NaAlg}$ film; (2) $70 \mathrm{wt} \% \mathrm{NaAlg}$, 30 wt\% glycerol (plasticizer) film; (3) Film 1: 57\% NaAlg, 24\% glycerol and 19\% PVPI; (4) Film 2: 52\% NaAlg, 22\% glycerol and 26\% PVPI; (5) Film 3:48\% NaAlg, 20\% glycerol and $32 \%$ PVPI and (6) pure PVPI complex film. 

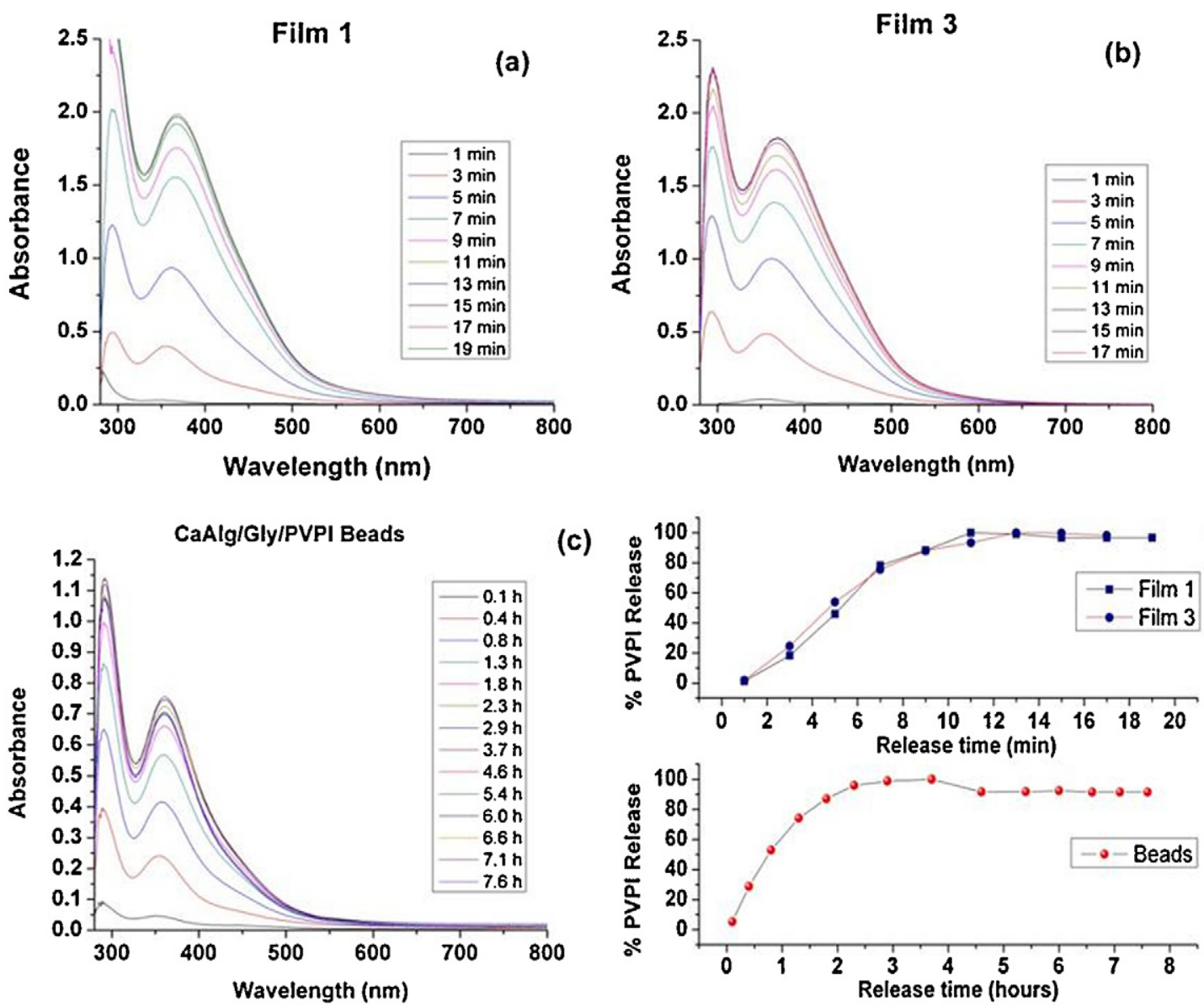

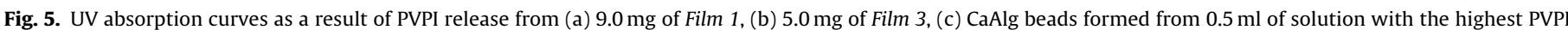
amount and (d) PVPI release kinetics in $2.5 \mathrm{ml}$ water.

by (3), (4), (5) and (6) and is attributed to the $C=0$ band stretching in PVPI complex. The shoulder at $1323 \mathrm{~cm}^{-1}$ was also only present in samples with PVPI and is due to aromatic amines present in the PVP molecule. Furthermore, the peak at 1296 is due to the $\mathrm{C}-\mathrm{O}$ stretch of the samples containing PVPI molecules. The peaks at 1100 and $1040 \mathrm{~cm}^{-1}$ are due to $\mathrm{C}-\mathrm{O}-\mathrm{C}$ anti-symmetric stretch and $\mathrm{C}-\mathrm{O}$ skeletal vibration of $\mathrm{C}-\mathrm{O}$ stretching (Kongjao, Damronglerd, \& Hunsom, 2010). We also observed that the higher the amount of the PVPI in NaAlg films, the higher the ratio of the intensity of PVPI associated carboxylate peak $\left(1665 \mathrm{~cm}^{-1}\right)$ to the NaAlg associated $1596 \mathrm{~cm}^{-1}$ carboxylate peak. In summary, the FTIR analysis indicates that there is no formation of new species when NaAlg and PVPI are blended in solution. This is an important conclusion since it demonstrates that upon physical mixing of the two polymers, NaAlg does not deteriorate or degrade the chemical constitution of the PVPI complex, so that PVPI can serve its purpose as a drug when embedded inside the NaAlg polymer matrix.

\subsection{Measurement of PVPI release from NaAlg films and CaAlg beads}

The dynamic release of the PVPI from the NaAlg films in water was investigated using UV absorption spectroscopy. Pieces of $9.0 \mathrm{mg}$ of Film 1 (18wt\% PVPI) and $5.0 \mathrm{mg}$ of Film 3 (32 wt\% PVPI), containing overall the same amount of PVPI, were cut and placed at the bottom of different UV cuvettes containing $2.5 \mathrm{ml}$ water each. Their UV absorption was recorded from the moment of their water immersion until the termination of release of the PVPI. According to literature, PVPI has two distinct absorption peaks due to the complexed $\mathrm{I}_{3}{ }^{-}$which are around 288 and $351 \mathrm{~nm}$ (Mazumdar, Chikindas, \& Ulrich, 2010). PVPI containing NaAlg films are expected to start swelling and then dissolve in water, releasing PVPI until the dissolution is completed. The measured absorbance intensity increases with time as more and more PVPI is released into water due to the swelling and subsequent dissolution of the NaAlg matrix (Fig. 5a and b). A small red swift of the two absorption peaks, and especially of the one at longer wavelengths, is noted, possibly due to the electron density changes caused by the complexation changes of the iodine ions and/or to conformational changes in the PVP polymer's backbone after its dissolution in water. The intensity of the second absorption peak was used for the evaluation of the PVPI release in water. The release dynamics are similar for the two films studied (first graph Fig. 5(d)) indicating that the PVPI concentration in the alginate films is not affecting its release. The absorption increase rate starts reducing after $7 \mathrm{~min}$, indicating approach of PVPI release termination. Within 11-13 min, the absorption intensity no longer increases indicating maximum PVPI release. Therefore, about $12 \mathrm{~min}$ are enough for the complete release in $2.5 \mathrm{ml}$ of water of a total amount of $1.60 \mathrm{mg}$ PVPI incorporated in NaAlg films, independently of the percentage concentration of the complex in the polymeric films.

Additionally, the release rate of encapsulated PVPI solution into freshly prepared CaAlg beads was also investigated in water. Beads obtained as described in Section 2.3, from approximately $0.5 \mathrm{~g}$ of the solution used for the preparation of Film 3 were immersed in the same cuvette used for the films, containing $2.5 \mathrm{ml}$ distilled water for UV absorption measurements. Fig. 5(c) shows the characteristic absorption spectra of the PVPI complex. It should be noted that 


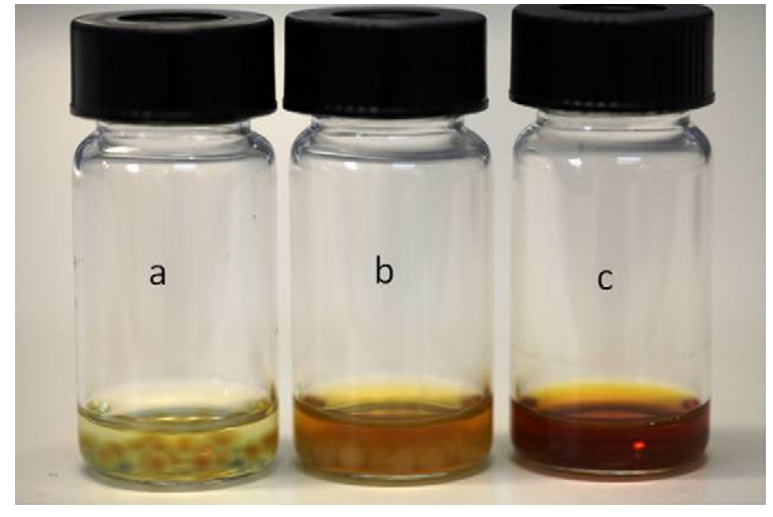

Fig. 6. (a) Beads, obtained from $0.5 \mathrm{ml}$ of Film 3 solution, placed inside a vial containing $2.5 \mathrm{ml}$ of water after $4 \mathrm{~h}$, (b) beads, obtained from $1 \mathrm{ml}$ of Film 3 solution, placed inside a vial containing $2.5 \mathrm{ml}$ of water after $4 \mathrm{~h}$ and (c) $0.5 \mathrm{ml}$ of Film 3 solution directly dispersed in $2.5 \mathrm{ml}$ water.

the red swift of the absorption peaks is quite reduced compared to the one monitored during the films' release. This indicates that the PVPI that is encapsulated in the CaAlg beads is already dissolved in water, and therefore the complexation of the iodine and/or the conformation of the PVP polymer's backbone should remain similar after its release. The intensity of the second absorption peak was used also in this case for the evaluation of the PVPI release in water. The release dynamics are represented in the second graph of Fig. 5(d), which shows that the release of PVPI in $2.5 \mathrm{ml}$ of water reaches a full plateau within $4 \mathrm{~h}$. The total amount of PVPI in the beads was calculated to be approximately $10 \mathrm{mg}$ (density of PVPI was taken as $1.0 \mathrm{~g} / \mathrm{ml}$ ). Note that the maximum absorption intensity of the PVPI released from the dissolving films is approximately twice than that released from the beads, even though the beads contain much more PVPI (10 mg) than the NaAlg films (1.60 mg). This observation indicates that at the end of the release procedure some PVPI should remain entrapped inside the CaAlg beads. To verify this assumption we performed the experiment presented if Fig. 6. In particular, Fig. 6(a) shows a photograph of CaAlg beads prepared from $0.5 \mathrm{ml}$ Film 3 solution, $4 \mathrm{~h}$ after they were placed inside a vial containing $2.5 \mathrm{ml}$ water. Fig. $6(\mathrm{~b})$, on the other hand, shows the state of the beads, which were obtained from $1 \mathrm{ml}$ of Film 3 solution, after $4 \mathrm{~h}$ in $2.5 \mathrm{ml}$ water. Finally, Fig. 6(c) shows a photograph of the $0.5 \mathrm{ml}$ Film 3 solution directly dispersed in $2.5 \mathrm{ml}$ of distilled water. Qualitative comparison of Fig. 6(a) and (c) indicates that the PVPI released from CaAlg beads in water after $4 \mathrm{~h}$ is clearly less than the one directly diluted in water using the same solution in amount and composition. This is due to some PVPI, which remains encapsulated inside the beads as clearly seen in Fig. 6(a). Even when the quantity of the beads, and thus the quantity of the entrapped PVPI, is doubled (Fig. 6b), there still appears to be less dissolved PVPI in the water compared to the one of the Film 3 solution directly dispersed in $2.5 \mathrm{ml}$ water. We propose that although the CaAlg bead formation is instant upon immersion of the NaAlg/PVPI droplets into the $\mathrm{CaCl}_{2}$ solution, it is possible that some of the PVPI may directly leach into the $\mathrm{CaCl}_{2}$ solution before or during the formation (cross linking) of the beads reducing the amount of encapsulated PVPI in the beads. Moreover, since iodine is complexed into PVP as $\mathrm{I}_{3}{ }^{-}$, calcium ions can easily form embedded $\mathrm{CaI}_{2}$ structures during alginate cross linking. In fact, this can be easily observed if some PVPI solution is added drop wise directly into excess $\mathrm{CaCl}_{2}$ solution, where the characteristic orange color of PVPI slowly turns colorless. $\mathrm{CaI}_{2}$ is a standard medicinal compound which is especially effective for bronchopulmonary diseases having also a partial antiseptic effect (Lopez-Belio, Henderson, Mansueto, \& Holinger, 1961). Therefore, formation of $\mathrm{CaI}_{2}$ is not considered as a drawback but as an added functionality opening up new application possibilities for these beads such as orally administered antiseptic and expectorant (cough, asthma, bronchitis and cystic fibrosis releaving agent). $\mathrm{CaI}_{2}$ is also an excellent diatery supplement (Berasategi et al., 2010).

\subsection{Antibacterial/antifungal activity of NaAlg/PVPI films and CaAlg/PVPI beads}

The antibacterial and antifungal activity of the composite films against $E$. coli and $C$. albicans was tested on films obtained by drop casting on two identical small glass slides which were placed

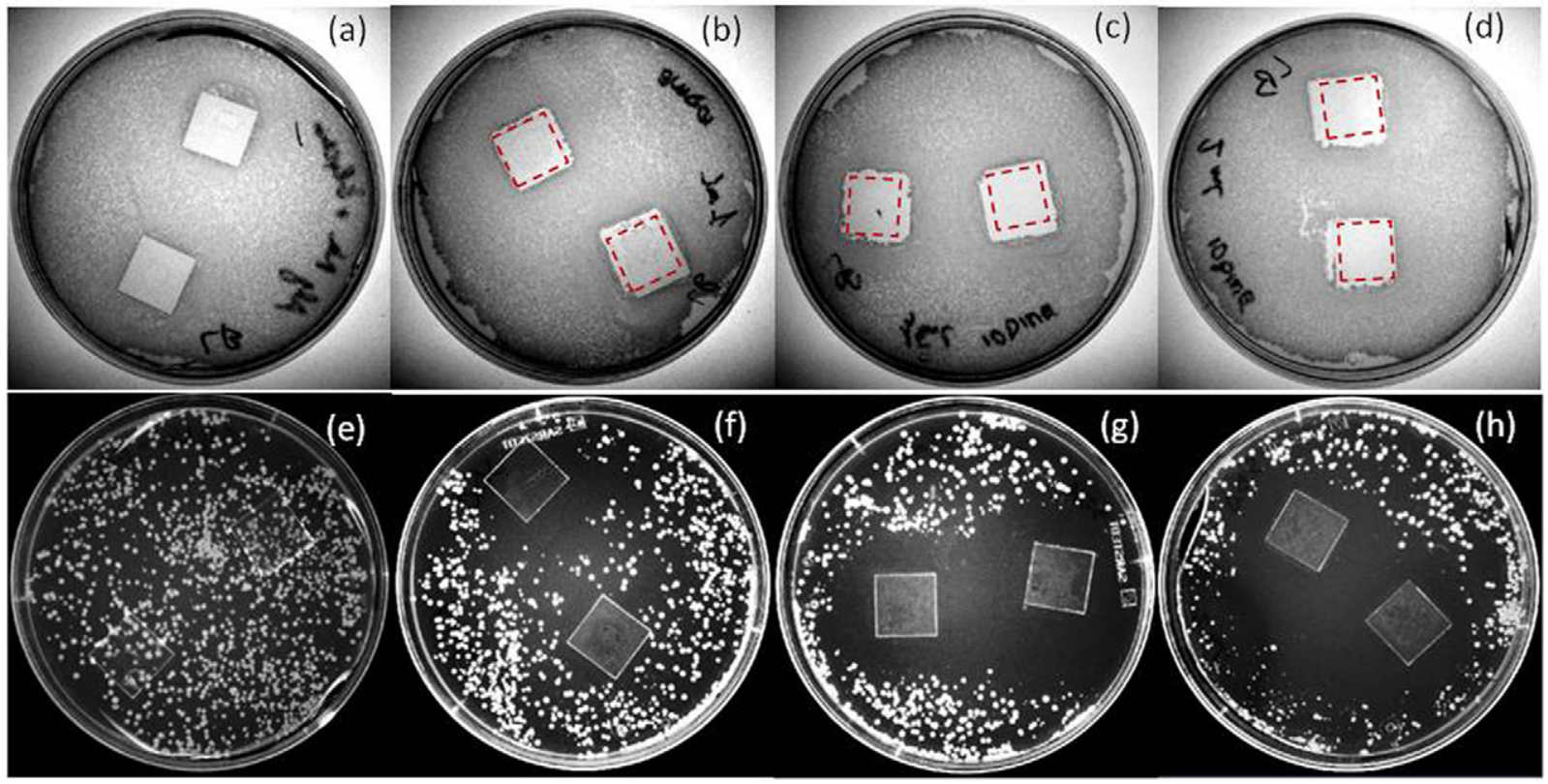

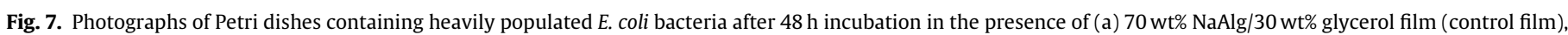

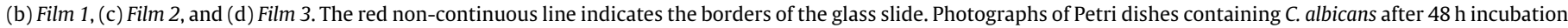

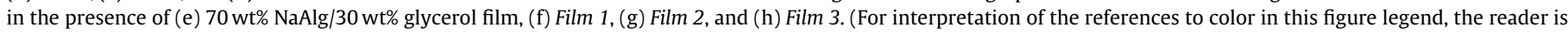
referred to the web version of this article.) 
(a)

\section{C. albicants}

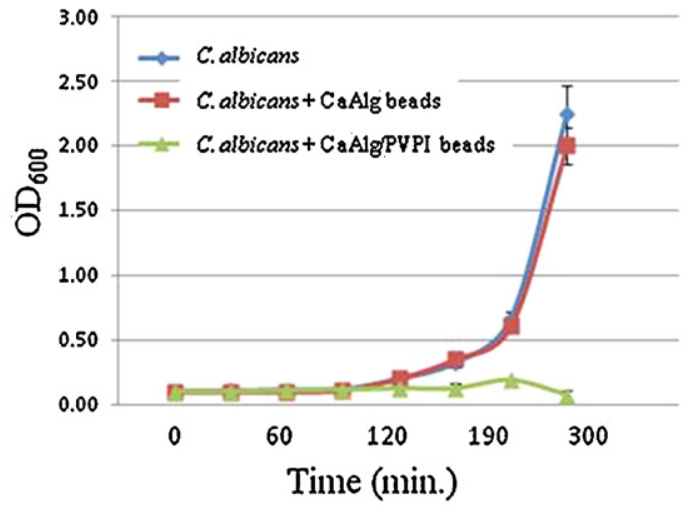

(b)

E. coli

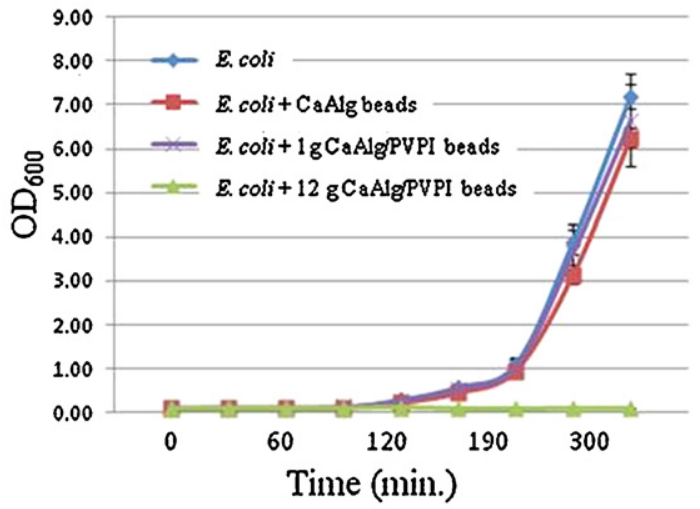

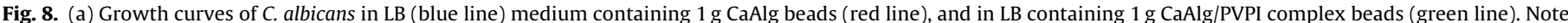

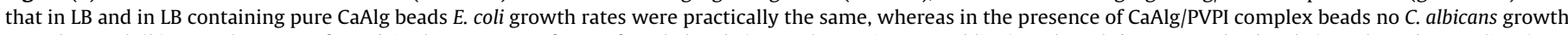

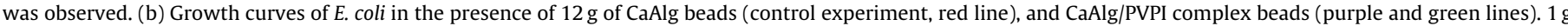

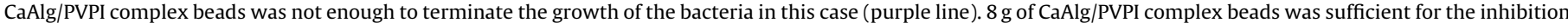
of bacteria growth (green line). (For interpretation of the references to color in this figure legend, the reader is referred to the web version of this article.)

in Petri dishes containing the bacterial or fungal media as seen in Fig. 7. For E. coli bacteria tests plastic Petri dishes were filled with Luria-Bertani agar medium. Fig. 7(a) shows the photograph of NaAlg/glycerol (control) film incubated in the heavily populated E. coli medium for $48 \mathrm{~h}$. At the end of the $48 \mathrm{~h}$ period, growth of bacteria all over the Petri dish was noticed. On the other hand, as seen in Fig. 7(b-d) corresponding to Films 1, 2 and 3, there was no $E$. coli growth on the coatings, and a thin inhibition zone free from bacteria was noticed around the films (the borders of which are shown with red dashed lines) after $48 \mathrm{~h}$ of incubation. The antibacterial effect of the three NaAlg/PVPI films was similar and there appeared to be no significant differences in bacteria growth inhibition between the films of lowest and highest PVPI concentration. For $C$. albicans tests, plastic Petri dishes were filled with SDA medium. The antifungal activity of the same films against C. albicans after $48 \mathrm{~h}$ of incubation in Petri dishes is reported in Fig. 7(e-h), respectively. The control film (NaAlg/glycerol) (Fig. 7e) did not show any antifungal activity. On the contrary, films containing PVPI complex inhibited the growth of $C$. albicans considerably. In fact, in this case, $24 \mathrm{~h}$ of incubation (not shown here) was sufficient to inhibit fungal growth with similar results to those obtained after $48 \mathrm{~h}$ incubation. These images clearly indicate that PVPI complex is highly active against fungal growth even if it is embedded in the NaAlg matrix. The formation of an inhibition zone around all films containing PVPI complex also indicates PVPI release from the NaAlg matrix, rendering these composites not only surface active but also prohibitive for fungal growth in their vicinity.

To assess the antibacterial and antifungal activity of CaAlg/PVPI beads against $E$. coli and $C$. albicans cells in water, we performed turbidity assays by detecting the spectral absorbance values at $600 \mathrm{~nm}$ of the cultures dispersed in water at different times. Absorbance at $600 \mathrm{~nm}$ is the generally recommended wavelength for measuring solutions containing $E$. coli or other bacteria (Glover, 1985; Harley \& Prescott, 2002). As shown in Fig. 8(a), the growth curves of $C$. albicans incubated with $1 \mathrm{~g}$ of antiseptic-free CaAlg beads are similar to the control experiment (without beads). On the other hand, the curve related to fungal growth with $1 \mathrm{~g}$ of NaAlg/PVPI beads clearly shows that the populations of cells do not increase at all, thus indicating cell death. Similar results were obtained with $E$. coli cultures as shown in Fig. 8(b), though a higher amount of CaAlg/PVPI beads $(12 \mathrm{~g})$ were needed to terminate the bacterial growth in this case. This is because the minimal bactericidal concentration (MBC) of PVPI was higher with respect to the minimal fungicidal concentration (MFC) (see Supplementary data).

\section{Conclusions}

Biodegradable NaAlg/PVPI composite films and $\mathrm{Ca}^{2+}$-crosslinked CaAlg/PVPI composite beads were prepared for the first time and their antimicrobial and antifungal activity was demonstrated. Alginate matrix enables time controlled release of PVPI, confirmed by UV spectra measurements, prolonging the well known PVPI antiseptic effect. Optical and AFM microscopy studies showed that PVPI complex forms circular micro-domains in the alginate matrix indicating micro-phase separation within the films. Detailed FTIR studies indicated that the blending process of the two materials does not cause chemical interactions thereby preserving PVPI's antiseptic properties. Moreover, cross linking NaAlg/PVPI blends with aqueous $\mathrm{CaCl}_{2}$ solutions enabled encapsulation of PVPI complex in CaAlg beads, which allow controlled release of the antiseptic in water saturated media. The most important potential application of these materials could be their use as external and internal wound dressings with controlled drug release properties. Their advantage toward the existing materials is that they are fully biocompatible and inexpensive. During surgery such beads can be easily used in open wounds without the need to remove them and without toxicity risks. Beads can be also incorporated in field waters as cost-effective disinfectants.

\section{Appendix A. Supplementary data}

Supplementary data associated with this article can be found, in the online version, at http://dx.doi.org/10.1016/j.carbpol. 2012.09.034.

\section{References}

Athanassiou, A., Bayer, I. S., Liakos, I., Rizzello, L., Cingolani, R., \& Pompa, P. P. (2012). Materiali compositi polimerici con proprietà antimicrobiche e biodegradabili e loro usi. Italy: Italian Patent Office Application no. TO2012A000258.

Berasategi, Z., Cuervo, M., Ruiz de las Heras, A, Santiago, S., Martinez, J. A., Astiasaran, I., et al. (2010). The inclusion of functional foods enriched in fibre, calcium iodine, fat-soluble vitamins and n-3 fatty acids in a conventional diet improves the nutrient profile according to the Spanish reference intake. Public Health Nutrition, 14(3), 451-458.

Blandino, A., Macias, M., \& Cantero, D. (1999). Formation of calcium alginate gel capsules: Influence of sodium alginate and $\mathrm{CaCl}_{2}$ concentration on gelation kinetics. Journal of Bioscience and Bioengineering, 88(6), 686-689.

Caykara, T., Demirci, S., Eroglu, M. S., \& Guven, O. (2006). Surface properties of binary blend films of poly(N-vinyl-2-pyrrolidone) and poly(vinyl alcohol) with sodium alginate. Journal of Polymer Science Part B: Polymer Physics, 44, 426-430. 
Chiu, C.-T., Lee, J.-S., Chu, C.-S., Chang, Y.-P., \& Wang, Y.-J. (2008). Development of two alginate-based wound dressings. Journal of Materials Science Materials in Medicine, 19, 2503-2513.

Cook, M. T., Tzortzis, G., Charalampopoulos, D., \& Khutoryanskiy, V. V. (2011). Production and evaluation of dry alginate-chitosan microcapsules as an enteric delivery vehicle for probiotic bacteria. Biomacromolecules, 12, 2834-2840.

Dantas, M. D. M., Cavalcante, D. R. R., Araújo, F. E. N., Barretto, S. R., Aciole, G. T. S., \& Pinheiro, A. L. B. (2011). Improvement of dermal burn healing by combining sodium alginate/chitosan-based films and low level laser therapy.Journal of Photochemistry and Photobiology B, 105, 51-59.

Donati, I., \& Paoletti, S. (2009). Material properties of alginates. In B. H. A. Rehm (Ed.), Alginates: Biology and applications (pp. 1-51). Berlin Heidelberg: Springer.

Glover, D. R. (Ed.). (1985). DNA cloning, a practical approach. Washington, DC: IRL Press.

Goh, C. H., Heng, P. W. S., \& Chan, L. W. (2012). Alginates as a useful natural polymer for microencapsulation and therapeutic applications. Carbohydrate Polymers, 88, $1-12$.

Heiner, J. D., Hile, D. C., Demons, S. T., \& Wedmore, I. S. (2010). 10\% Povidoneiodine may be a practical field water disinfectant. Wilderness and Environmental Medicine, 21(4), 332-336.

Kongjao, S., Damronglerd, S., \& Hunsom, M. (2010). Purification of crude glycerol derived from waste used-oil methyl ester plant. Korean Journal of Chemical Engineering, 27(3), 944-949.

Lakhal, K., Faidherbe, J., Choukhi, R., Boissier, E., \& Capdevila, X. (2011). Povidone iodine: Features of critical systemic absorption. Annales Françaises d'Anesthésie et de Réanimation, 30(7-8), e1-e3.

Lawrie, G., Keen, I., Drew, B., Chandler-Temple, A., Rintoul, L., \& Fredericks, P. (2007). Interactions between alginate and chitosan biopolymers characterized using FTIR and XPS. Biomacromolecules, 8(8), 2533-2541.

Lopez-Belio, M., Henderson, W. J., Mansueto, W. J., \& Holinger, M. P. H. (1961). The concentration of I131 in the bronchial tree after oral administration of tagged $\mathrm{CaI}_{2}$ and $\mathrm{KI}$. Chest, 39(2), 158-161.

Maizura, M., Fazilah, A., Norziah, M. H., \& Karim, A. A. (2007). Antibacterial activity and mechanical properties of partially hydrolyzed sago starch-alginate edible film containing lemongrass oil. Journal of Food Science, 72(6), C324-C330.

Mazumdar, N., Chikindas, M. L., \& Ulrich, K. (2010). Slow release polymer-iodine tablets for disinfection of untreated surface water. Journal of Applied Polymer Science, 117, 329-334.

Mishra, A., \& Chaudhary, N. (2010). Study of povidone iodine loaded hydrogels as wound dressing material. Trends in Biomaterials \& Artificial Organs, 23(3), $122-128$.
Moe, S. T., Draget, K. I., Skjak-Bræk, G., \& Smidsrod, O. (1995). Food polysaccharides and their applications. In A. M. Stephen (Ed.), Alginates (pp. 245-286). New York: Marcel Dekker.

Nahlielia, O., Baruchinb, A. M., Levia, D., Shapiraa, Y., \& Yoffea, B. (2001). Povidoneiodine related burns. Burns, 27, 185-188

Paños, I., Acosta, N., \& Heras, A. (2008). New drug delivery systems based on chitosan. Current Drug Discovery Technologies, 5, 333-341.

Patel, G., Patel, G., Patel, R., Patel, J., Bharadia, P., \& Patel, M. (2007). Sodium alginate: Physiological activity, usage \& potential applications. Drug Delivery Technology, 7(4), 28-37.

Harley, J. P., \& Prescott, L. M. (5th Eds.). (2002). Laboratory exercises in microbiology. New York: The McGraw-Hill Companies.

Russo, R., Malinconico, M., Petti, L., \& Romano, G. (2005). Physical behavior of biodegradable alginate-poly(vinylalcohol) blend films. Journal of Polymer Science Part B: Polymer Physics, 43, 1205-1213.

Sachan, N. K., Pushkar, S., Jha, A., \& Bhattcharya, A. (2009). Sodium alginate: The wonder polymer for controlled drug delivery. Journal of Pharmacy Research, 2(8), 1191-1199.

Sartori, C., Finch, D. S., Ralph, B., \& Gilding, K. (1997). Determination of the cation content of alginate thin films by FTIR spectroscopy. Polymer, 38(1) 43-51.

Shilpa, A., Agrawal, S. S., \& Ray, A. R. (2003). Controlled delivery of drugs from alginate matrix. Journal of Macromolecular Science Polymer Reviews, C43, 187-221.

Sikareepaisana, P., Ruktanonchai, U., \& Supaphol, P. (2011). Preparation and characterization of asiaticoside-loaded alginate films and their potential for use as effectual wound dressings. Carbohydrate Polymers, 83, 1457-1469.

Simmons, T. J., Lee, S.-H., Park, T.-J., Hashim, D. P., Ajayan, P. M., \& Linhardt, R. J. (2009). Antiseptic single wall carbon nanotube bandages. Carbon, 47, 1561-1564.

Spano, F., Massaro, A., Blasi, L., Malerba, M., \& Cingolani, R. A. A. (2012). In situ formation and size control of gold nanoparticles into chitosan for nanocomposite surfaces with tailored wettability. Langmuir, 28(8), 3911-3917.

Wang, L., Khor, E., Wee, A., \& Yong Lim, L. (2002). Chitosan-alginate PEC membrane as a wound dressing: Assessment of incisional wound healing. Journal of Biomedical Materials Research, 63(5), 610-618.

Wee, S., \& Gombotz, W. R. (1998). Protein release from alginate matrices. Advanced Drug Delivery Reviews, 31(3), 267-285.

Wong, R. H. L., Wong, V. W. Y., Hung, E. C. W., Lee, P.-Y., Ng, C. S. H., \& Wan, I. Y. P. (2011). Topical application of povidone-iodine before wound closure is associated with significant increase in serum iodine level. Surgical Practice, 15(3), 79-82. 